\title{
lon charge number and flux saturation effects in the corona of a laser- irradiated pellet
}

J. Sanz and J.R. Sanmartín

E. T. S. Ingenieros Aeronáuticos, Universidad Politécnica de Madrid, Madrid-3, Spain

(Received 29 December 1981; accepted 29 July 1983)

\begin{abstract}
The quasisteady structure of the corona of a laser-irradiated pellet is completely determined for arbitrary $Z_{l}$ (ion charge number) and $r_{c} / r_{a}$ (ratio of critical and ablation radii), and for heat-flux saturation factor $f$ above approximately 0.04 . The ion-to-electron temperature ratio at $r_{c}$ grows sensibly with $Z_{i}$; all other quantities depend weakly and nonmonotonically on $Z_{t}$. For $r_{c} / r_{a}$ close to unity, and all $Z_{t}$ of interest $\left(Z_{t}<47\right)$, the flow is subsonic at $r_{c}$. For a given laser power $W$, flux saturation may decrease (low $f$ ) or increase (high $f$ ) the ablation pressure $P_{a}$ relative to the value obtained when saturation is not considered; in some cases a decrease in $f$ with $W$ fixed increases $P_{a}$. For intermediate $f(\sim 0.1), P_{a} \propto\left(W / r_{a}^{2}\right)^{2 / 3} \rho_{c}^{1 / 3}\left(\rho_{c}=\right.$ critical density $)$, independently of $r_{c} / r_{a}$; for $f \sim 0.6, P_{a}$ is larger by a factor of about $\left(r_{c} / r_{a}\right)^{2 / 3}$. For $r_{c} / r_{a}>1.2$ roughly, the mass ablation rate is $C\left(Z_{t}\right)\left[\left(m_{t} / k Z_{t}\right)^{7 / 2} \bar{K} r_{a}^{11} P_{a}^{5}\right]^{1 / 6}$, independent of $\rho_{c}$ and $f$, and barely dependent on $Z_{t}\left(m_{l}\right.$ is ion mass; $k$, Boltzmann's constant; $\bar{K}$, conductivity coefficient; and $C$, a tabulated function).
\end{abstract}

\section{INTRODUCTION}

The complex set of phenomena involved in laser fusion is usually studied through thorough numerical simulations. It is clear, however, that simple scaling laws for partial aspects of the entire process would be quite interesting. In the recent past there have been attempts to derive such laws for the corona of plasma ablated from the target, setting aside the problem of target compression. Reasonable approximations introduced in the analysis have been (a) fluid description, (b) spherically symmetric flow, (c) quasisteady flow, (d) classical (Spitzer's) conductivity, and (e) anomalous absorption at the critical density $n_{c}$ (negligible inverse bremsstrahlung). In the analyses relations were sought between laser power $W$, wavelength $\lambda$ (or $n_{c}$ ), instantaneous pellet radius $r_{a}$, average ion mass $m_{l}$ and charge number $Z_{l}$, pressure at the cold ablation surface $P_{a}$, ablation rate constant $\mu$, radius of critical surface $r_{c}$, temperatures $T_{e c}$ and $T_{t c}$, etc.

The problem was first discussed by Caruso $^{1}$ who suggested that, for $r_{c} / r_{a} \rightarrow \infty$, the critical density $n_{c}$, then lying at infinity, would be an ignorable parameter. Later Gitomer et $a .^{2}$ and Afanas'ev et al. ${ }^{3}$ carried out detailed analytical studies. Max et al. ${ }^{4}$ introduced into the problem the usual flux-limit factor $f$ to take into account that flux saturation must occur over part of the corona under a broad range of conditions; they presented a thorough discussion of physical phenomena not included in the analysis. Saturation in a related astrophysical context had been discussed by Cowee and McKee. ${ }^{5}$ Nuckolls ${ }^{6}$ had earlier suggested how flux saturation would affect the relation $P_{a}(W)$.

The analysis of Refs. 2 and 3 was reexamined by the authors elsewhere. ${ }^{7}$ If $r_{a}, n_{c}, m_{t}$, and $Z_{t}$ are given, their dimensionless formulation leads to a two-eigenvalue problem, in which $r_{c} / r_{a}$ is a free parameter in the range $(1, \infty)$. One then obtains complete results for $W, P_{a}, \mu$, etc., in dimensionless form. In particular, elimination of $r_{c} / r_{a}$ yields $W$ and $\mu$ in terms of $r_{a}, P_{a}, n_{c}, m_{i}$, and $Z_{i}$; briefly $W\left(r_{a}, P_{a}\right)$ and $\mu\left(r_{a}, P_{a}\right)$. The interest of this is that the "inner" problem (the region $r<r_{a}$, as opposed to the outer one, $r>r_{a}$ ) can in principle be solved, if $\mu\left(r_{a}, P_{a}\right)$ is known, for any kind of compression desired, without further consideration of the corona; in each case one would then obtain laws $r_{a}(t), P_{a}(t)$, and thus finally $\mu(t)$ and $W(t)$.

The formulation of Ref. 7 showed that the behavior of the far away plasma evolves as $W$ increases $\left(T_{e}\right.$ first decaying with distance as $r^{-4 / 3}$, then as $r^{-2 / 5}$, and finally as $\left.r^{-2 / 7}\right)$, with a dramatic effect on the relation $P_{a}(W)$ : When $r_{c} / r_{a}$ (or $W$ ) is large, the absorbed laser energy is mostly conducted outward, leading to an ablation pressure well below that predicted by Caruso'; for $r_{c} / r_{a}$ large, $n_{c}$ is not an ignorable parameter. This effect went unnoticed in Ref. 2, where no explicit results were given, and in Ref. 3, where wrong laws were presented $\left(P_{a} \propto W^{1 / 2} / r_{a}^{7 / 4}, T_{e c} \propto P_{a}^{2 / 3} r_{a}^{3 / 4}\right.$, etc., for $r_{c} / r_{a}$ not too close to unity). Again the special behavior of the solution for $r_{c} / r_{a}$ close to unity was either unnoticed ${ }^{2}$ or incorrectly determined ${ }^{3}$ because the structure of a laser-produced deflagration layer was not taken into account. Finally, Ref. 7 showed that one should let $T_{e} \neq T_{1}$ for consistency (times for ion-electron energy relaxation and electron heat conduction being comparable); $T_{t}$ was decoupled from the problem by assuming $Z_{t}$ large, and correctly determined at the end. Gitomer et al. ${ }^{2}$ had set $T_{e}=T_{t}$; Afanas'ev et al. ${ }^{3}$ assumed $Z_{l}$ large but their $T$, was wrong because they did not take into account ion cooling due to expansion.

In the present work we extend the analysis of Ref. 7 to include finite (arbitrary) $Z$, values (Sec. II) and flux saturation (Sec. III). In the final discussion of results (Sec. IV) we include a comparison with the analysis and results of Refs. 4 and 6. 


\section{ARBITRARY ION CHARGE NUMBER}

Following the earlier work of Sanz et al., ${ }^{7}$ we consider the equations for quasineutral, steady, spherically symmetric flow: mass conservation

$$
n v r^{2}=\mu \text {, }
$$

ion-electron momentum conservation

$$
m_{\imath} n v \frac{d v}{d r}=-\frac{d}{d r} n k\left(Z_{t} T_{e}+T_{\imath}\right),
$$

ion entropy

$$
\frac{n}{Z_{\iota}} T_{\iota} v \frac{d}{d r}\left(k \ln \frac{T_{\iota}^{3 / 2}}{n}\right)=\frac{3}{2} k n^{2} \frac{T_{e}-T_{t}}{\bar{t}_{e l} T_{e}^{3 / 2}},
$$

and an overall energy equation which, making use of the boundary conditions at $r_{a}\left(v=T_{e}=T_{t}=T_{e}^{5 / 2} d T / d r=0\right)$, reads

$$
\begin{aligned}
& \mu\left(\frac{5}{2} k\left(Z_{t} T_{e}+T_{l}\right)+\frac{1}{2} m_{t} v^{2}\right) \\
& =Z_{t} r^{2} \bar{K} T_{e}^{5 / 2} \frac{d T_{e}}{d r}+\frac{W Z_{t} H(r)}{4 \pi}, \\
& H=0 \quad\left(r<r_{c}\right), \quad H=1 \quad\left(r>r_{c}\right),
\end{aligned}
$$

and is used instead of the entropy equation for electrons. All symbols have the usual meaning.

Defining

$$
\begin{aligned}
& \eta=\frac{r}{r_{a}}, \theta_{e}=\frac{T_{e}}{T_{r}}, \theta_{i}=\frac{T_{t}}{T_{r}}, \\
& u=\frac{v}{\left(Z_{t} k T_{r} / m_{l}\right)^{1 / 2}},
\end{aligned}
$$

and choosing $T_{r}$ in such a way that the boundary condition $P\left(r_{a}\right)=P_{a}$, using (1), reads

$$
u / \theta_{e}=1 \text { at } \eta=1 \text {, }
$$

we find

$$
T_{r}=\left(\frac{Z_{t}}{1+Z_{t}}\right)^{2} \frac{Z_{t}}{m_{t} k} \frac{P_{a}^{2} r_{a}^{4}}{\mu^{2}}
$$

and

$$
\begin{aligned}
\frac{1}{2}(1 & \left.-\frac{\theta_{e}+5 \theta_{t} / 3 Z_{t}}{u^{2}}\right) \frac{d u^{2}}{d \eta} \\
& =\frac{2}{\eta}\left(\theta_{e}+\frac{5 \theta_{t}}{3 Z_{t}}\right)-\frac{d \theta_{e}}{d \eta}-\frac{6.45 b}{\beta Z_{t}} \frac{\theta_{e}-\theta_{t}}{\eta^{2} u^{2} \theta_{e}^{3 / 2}},
\end{aligned}
$$

$\frac{5}{2}\left(\theta_{e}+\frac{\theta_{t}}{Z_{t}}\right)+\frac{1}{2} u^{2}=\beta \eta^{2} \theta_{e}^{5 / 2} \frac{d \theta_{e}}{d \eta}+\bar{W} H(\eta)$,

$\beta \eta^{2}\left(\frac{3}{2} u^{2} \frac{d \theta_{t}}{d \eta}+\frac{2 u^{2} \theta_{t}}{\eta}+\frac{1}{2} \theta_{t} \frac{d u^{2}}{d \eta}\right)$

$$
=9.68 b \frac{\theta_{e}-\theta_{t}}{\theta_{e}^{3 / 2}},
$$

$H=0 \quad\left(\eta<\eta_{c}\right), \quad H=1 \quad\left(\eta>\eta_{c}\right)$.

Here

$$
\begin{aligned}
& \beta=\left(\frac{Z_{t}}{1+Z_{1}}\right)^{5}\left(\frac{Z_{1}}{m_{1}}\right)^{5 / 2} \frac{\bar{K}}{k^{7 / 2}} \frac{r_{a}^{11} P_{a}^{5}}{\mu^{6}}, \\
& \bar{W}=\left(\frac{Z_{\imath}+1}{Z_{\iota}}\right)^{2} \frac{m_{\imath}}{4 \pi Z_{\iota}} \frac{\mu W}{P_{a}^{2} r_{a}^{4}}, \\
& \eta_{c}^{2} u\left(\eta_{c}\right)=\frac{Z_{t}+1}{Z_{\imath}} \frac{m_{\imath}}{Z_{t} n_{c}} \frac{\mu^{2}}{P_{a} r_{a}^{4}} ;
\end{aligned}
$$

$b\left(Z_{i}\right) \equiv Z_{i} \bar{K}\left(Z_{l}\right) / \bar{K}(1)$ varies between 1 at $Z_{i}=1$ and 4.2 for $Z_{\imath} \rightarrow \infty$.

The boundary conditions are (5) and

$$
\begin{aligned}
& \theta_{e}=0, \quad \theta_{1} / \theta_{e}=1 \text { at } \eta=1, \\
& \theta_{e} \rightarrow 0, \quad \text { as } \eta \rightarrow \infty,
\end{aligned}
$$

together with either one of two conditions,

$$
\frac{d \theta_{e}}{d \eta}=2 \frac{\theta_{e}+5 \theta_{i} / 3 Z_{t}}{\eta}-\frac{6.45 b}{\beta Z_{i}} \frac{\theta_{c}-\theta_{i}}{\eta^{2} u^{2} \theta_{e}^{3 / 2}}
$$

or

$$
\eta_{c}=\eta_{s},
$$

at the point $\eta_{s}$ where $u^{2}=\theta_{e}+5 \theta_{1} / 3 Z_{r}$. Notice that conditions (12), required to avoid multivalued solutions in Eq. (6), occur at a sonic point, partly isothermal, partly isentropic.

As in Ref. 1 we solve Eqs. (6)-(8) choosing as free parameter $\eta_{c}$, in the range $1<\eta_{c}<\infty$; for each value we find $\beta$, $\bar{W}$, and $u\left(\eta_{c}\right)$, and then through $(9)-(11)$ we arrive at $P_{a}, W$, and $\mu$. Now, however, $T_{t}$ is coupled to both $v$ and $T_{e}$.

For $\eta<\eta_{c}(H=0)$, we may introduce phase-space variables $Y \equiv \beta \eta \theta_{e}^{5 / 2}, M^{2} \equiv u^{2} / \theta_{e}$, together with $\psi \equiv \theta_{t} / \theta_{e}$; they obey equations

$$
\begin{aligned}
& \frac{d Y}{d \ln \eta}=F_{1}(M, \psi, Y), \\
& \frac{d M^{2}}{d Y}=F_{2}(M, \psi, Y), \quad \frac{d \psi}{d Y}=F_{3}(M, \psi, Y) .
\end{aligned}
$$

Assume first that the flow, which starts as subsonic $\left(u^{2}\right)$ $\theta \simeq u \simeq 0$ at $\eta=1)$, is supersonic at $\eta_{c}\left(\eta_{s}<\eta_{c}\right)$. Condition (12a) shows that the solution must cross the sonic plane $\pi_{s}$,

$$
M^{2}=1+5 \psi / 3 Z
$$

at its intersection $L_{0}$ with the surface

$$
2 M^{2}(Y-1)-1=\left(6.45 b / Z_{l}\right) \frac{(1-\psi)}{M^{2}} ;
$$

$L_{0}$ is a line of saddle points. The solution to (14) near ts singular point $Y=0, M^{2}=0, \psi=1$, which is part node, part saddle and corresponds to $\eta=1$, takes the form

$$
M^{2} \simeq\left(\frac{Y}{\beta}\right)^{2 / 5}, \quad \psi-1=\frac{25\left(Z_{t}+1\right)}{6 \times 6.45 b Z_{t}}\left(\frac{Y}{\beta}\right)^{2 / 5} ;
$$

this one-parameter family of curves intersects $\pi_{s}$ in a curve $L_{s}$. Sweeping through positive values of $\beta$ we determine $L_{s}$, and thus the value $\beta_{l}$ for which it meets $L_{0}$ at a point $Y_{s l}, M_{s l}, \psi_{s l}$. The topology of the solutions is shown in Fig. 1: $L_{s}$ ends at $L_{0}$; for $\beta>\beta_{l}$ the solutions do not reach $\pi_{s}$ and the flow stays everywhere subsonic.

Integrating from the point $Y_{s l}, M_{s l}, \psi_{s l}$ into the supersonic half-space, we entirely determine $M^{2}(Y), \psi(Y)$. Then 


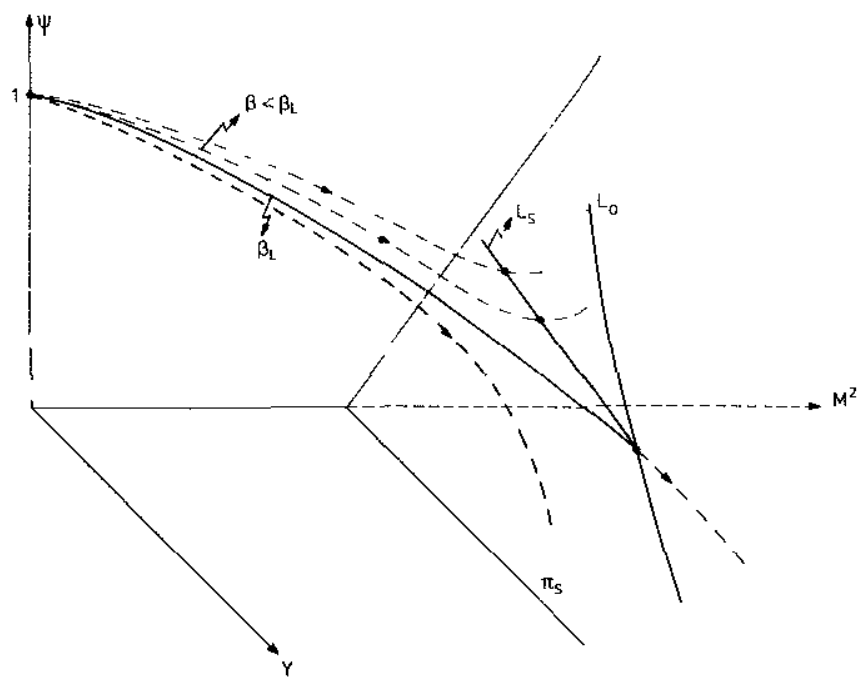

FIG. 1. Topology of integral curves in the phase space of Eqs. (14).

Eq. (13), with the boundary condition $Y=0$ at $\eta=1$, yields the sonic radius $\eta_{s l}$ and $Y(\eta)$, and hence $u(\eta), \theta_{e}(\eta)$, and $\theta_{i}(\eta)$ (shown in Fig. 2 for $Z_{i}=1$ ) up to the critical radius $\eta_{c}$, wherever it may be. For any chosen $\eta_{c}>\eta_{s l}$, all three values $u\left(\eta_{c}\right)$, $\theta_{e}\left(\eta_{c}\right)$, and $\theta_{i}\left(\eta_{c}\right)$ are known. Equations $(6)-(8)$ could be integrated, starting at $\eta_{c}$, if $\bar{W}$ were known. The quantity $\bar{W}$ is then determined by the condition at infinity $\left(\theta_{e} \rightarrow 0\right)$.

As $\bar{W}$ (and $\eta_{c}$ ) decreases a value $\bar{W}_{l}$ is reached for which $\eta_{c}=\eta_{s l} ;$ both Eqs. $(12 \mathrm{a})$ and $(12 \mathrm{~b})$ are then satisfied. There are two slopes at $\eta_{c}$ : Equation (12a) holds clearly at $\eta_{c}^{-}\left(\eta_{s}=\eta_{c}^{-}\right)$. For $\bar{W}_{<} \bar{W}_{l}$ (or $\left.\eta_{c}<\eta_{s l}\right)$ Eq. (12b) must be used instead of (12), that is, the solution corresponds to some value $\beta<\beta_{1}$. For any such $\beta$, integration of (13) and (14) yields $\eta_{,}, u\left(\eta_{s}\right), \theta_{c}\left(\eta_{s}\right)$, and $\theta_{1}(\eta$,$) . Since \eta_{c}=\eta_{s}$, to solve for the range $\eta>\eta_{s}$ one proceeds as previously.

As $\eta_{c}$ decreases further a value $\eta_{c s}$ may be found such that Eq. (12a), in addition to (12b), is again satisfied; now (12a) holds at $\eta_{c}^{+}\left(\eta_{s}=\eta_{c}^{+}\right)$. For $1<\eta_{c}<\eta_{c s}$, Eq. (12a) and not (12b) must be used $\left(\eta_{s}>\eta_{c}\right)$. At $\eta_{s}$ (and in general for any $\eta>\eta_{c}$ ) one should have $d \theta / d \eta<0$. This is impossible for $Z_{i} \rightarrow \infty$, when the last term in (12a) vanishes ${ }^{7}$; thus, no such $\eta_{c s}$ exists for $Z_{i}$ above some value, which we find numerically to be approximately 46 .

As $\eta_{c} \rightarrow 1$, we find $\beta \rightarrow 0$; heat conduction is then limited to a thin deflagration layer (adjoining the pellet) which involves large gradients and may be treated as planar. ${ }^{7}$ Outside, the flow is isentropic and $T_{i}=T_{e}$.

Figures 3 and 4 show $P_{a}$ vs $W$, and $\mu$ vs $P_{a}$, respectively; Fig. 5 gives $r_{c} / r_{a}, P\left(r_{c}\right) / P_{a}$, and $T_{t}\left(r_{c}\right) / T_{e}\left(r_{c}\right)$ vs $P_{a}$. Figures 6 and 7 relate $W$ to $P_{a}$ and mass ablation rate $(\dot{m}$ $\left.\equiv 4 \pi m_{i} \mu / Z_{i}\right)$, and to $T_{e}\left(r_{c}\right)$ and $T_{i}\left(r_{c}\right)$, respectively, for particular conditions. Figure 8 and Table I give $r_{s}\left(r_{c}\right)$, and $\beta_{l}$ and $\eta_{c}^{*},{ }^{8}$ respectively, for several values of $Z_{i}$.

\section{HEAT FLUX SATURATION EFFECTS}

When the electron mean free path is larger than the characteristic length for temperature changes, the classical result, $q=-\bar{K} T_{e}^{5 / 2} d T_{c} / d r$, yields unphysically large values of the heat flux. A simple way to correct for this has been to saturate, or limit the value of, $q$, in the form

$$
|q| \leqslant f n k T_{c}\left(k T_{c} / m_{e}\right)^{1 / 2} .
$$

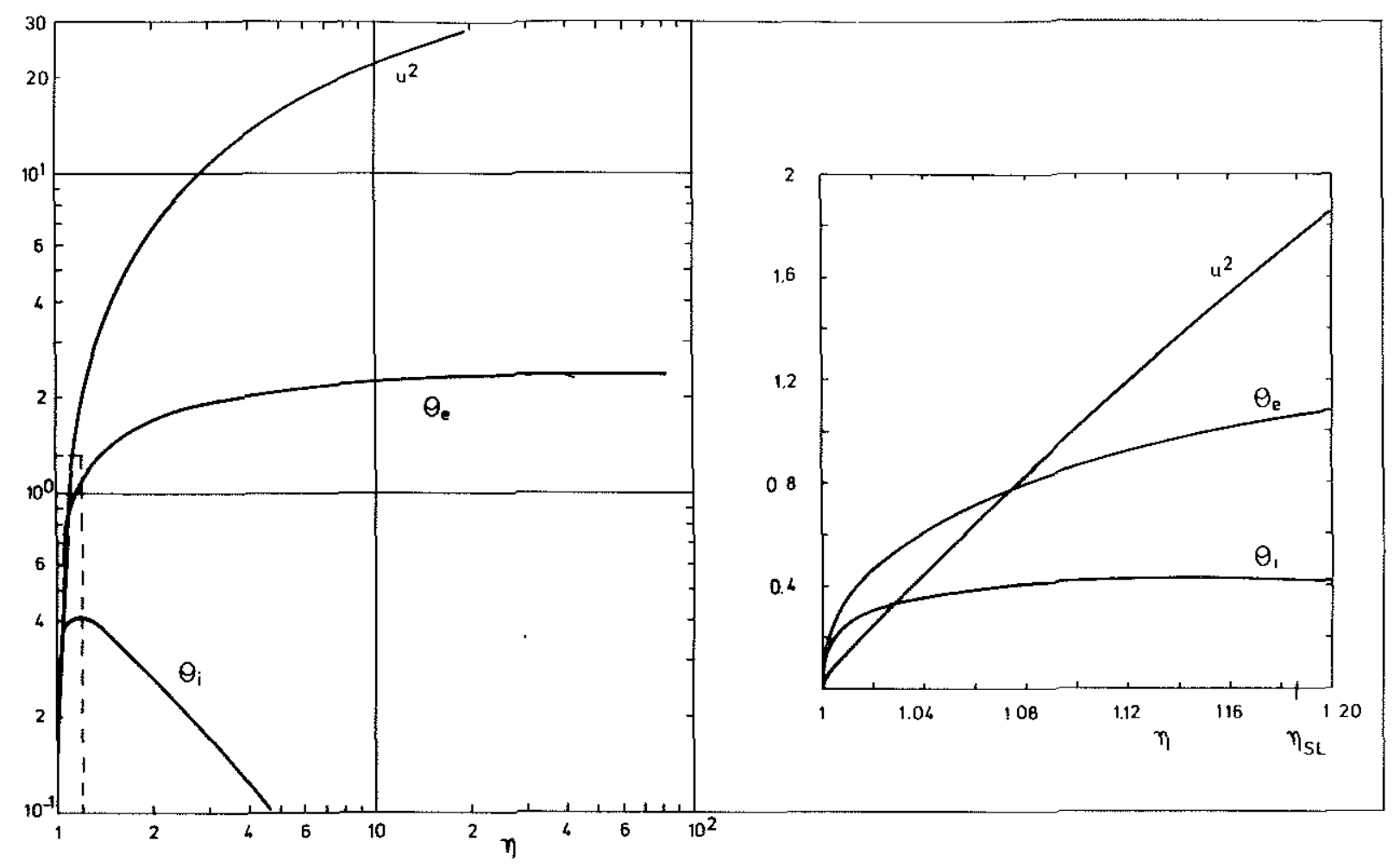

FIG. 2. Dimensionless squared velocity and temperatures versus radius $\left(\eta \equiv r / r_{a}\right)$ for $1<\eta<\eta_{c}$ and $\eta_{s t}<\eta_{c}$, and $Z$, $=1$. 


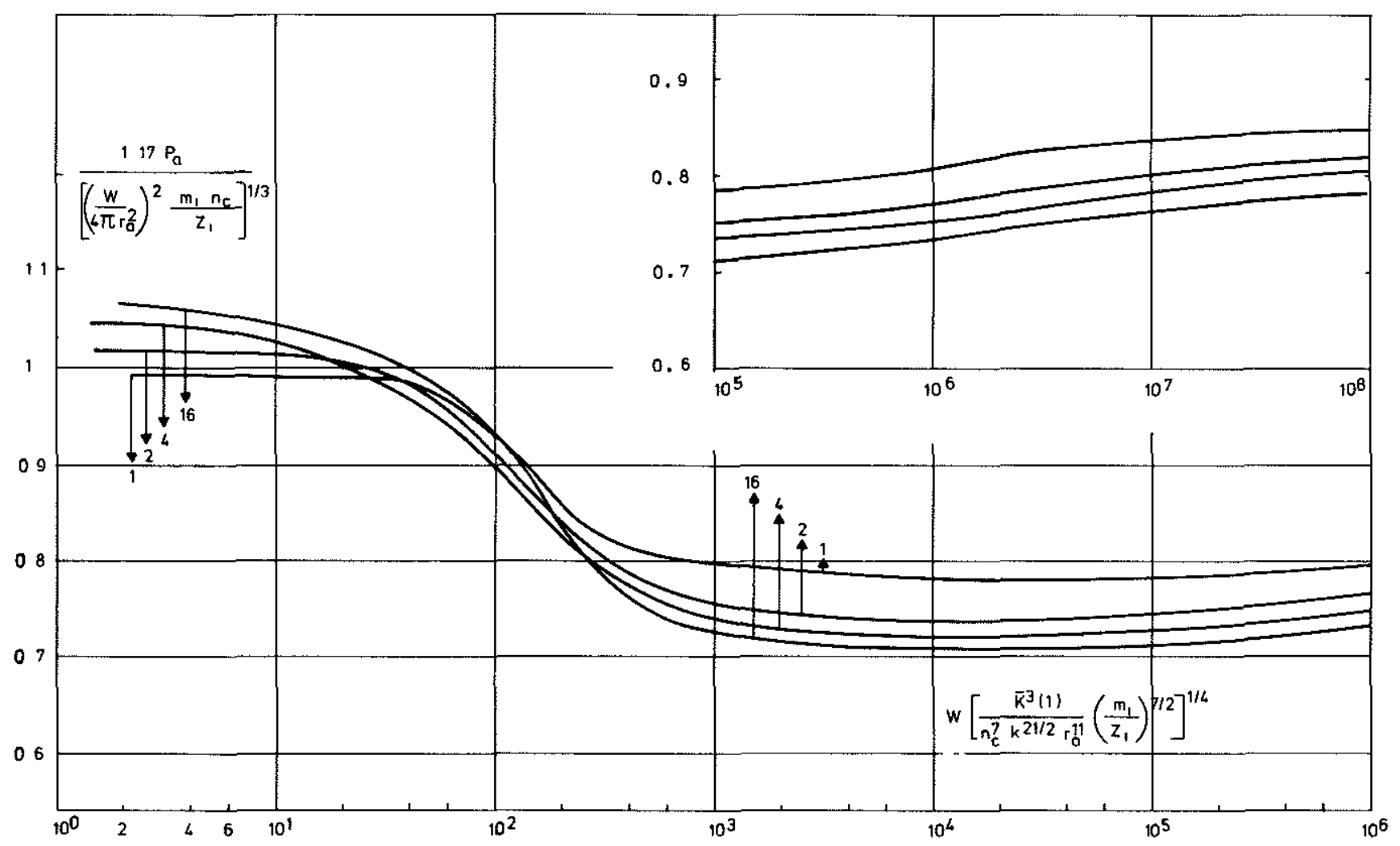

FIG. 3. Ablation pressure $P_{a}$ versus absorbed laser power $W$, for $Z,=1,2,4$, and 16 . In convenient units, the horizontal axis is $39.7 W \lambda^{7 / 2}\left(A, / Z_{t}\right)^{7 / k} r_{a} 11 / 4$ and the vertical axis is $0.522 P_{a} r_{a}^{4 / 3} \lambda^{2 / 3} W^{-2 / 3}\left(Z, / A_{t}\right)^{1 / 3} ; P_{a}$ is measured in $10^{6}$ bars, $W$ in $10^{12} W, \lambda$ in $\mu \mathrm{m}$, and $r_{a}$ in mm. Here $A$, is the ion mass number.

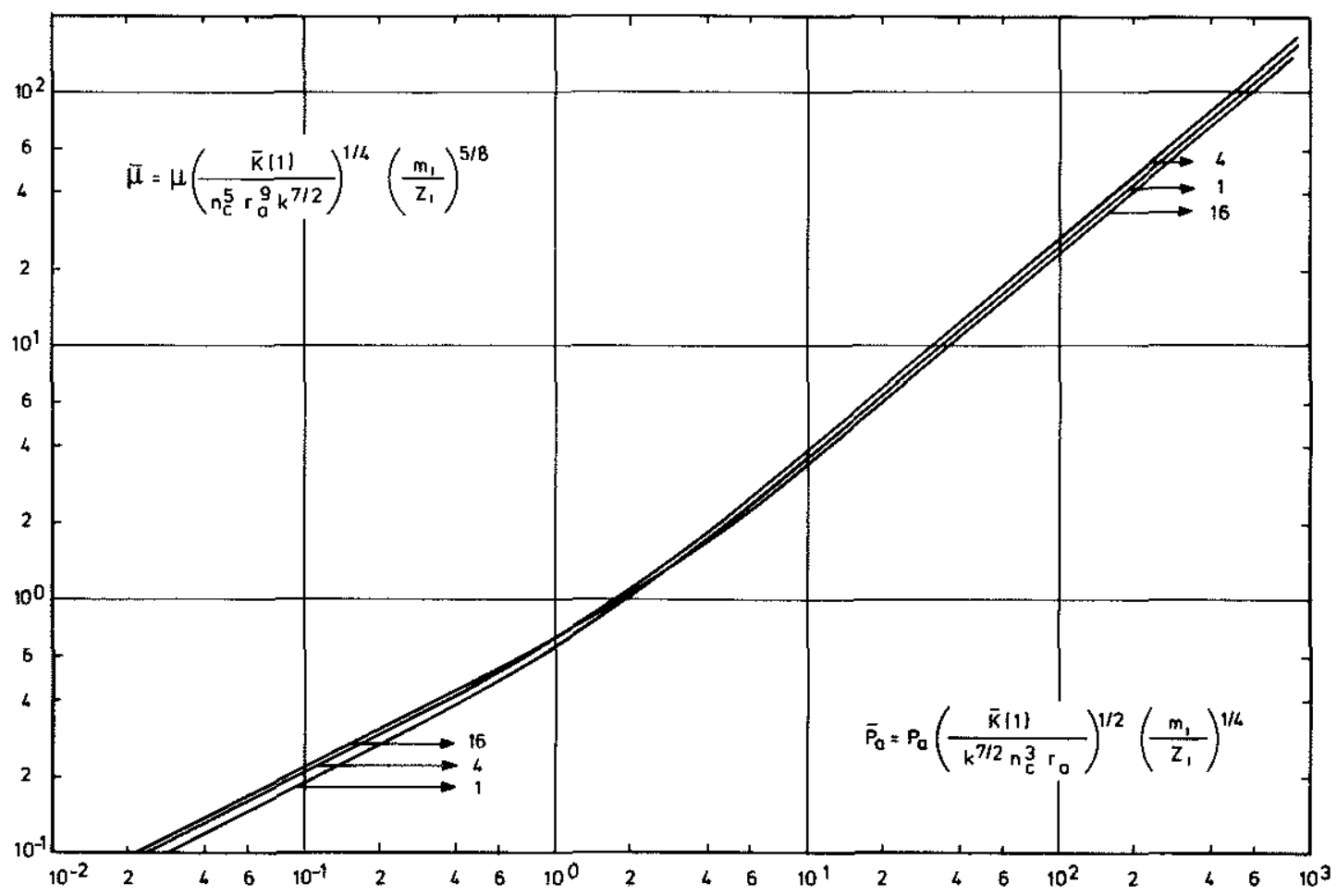

FIG. 4. Mass ablation rate $\dot{m}$ vs $P_{a}$ for $Z_{l}=1,4$, and 16 . The horizontal axis is $0.961 P_{a} \lambda^{3}\left(A_{l} / Z_{l}\right)^{1 / 4} r_{a}{ }^{1 / 2}$ and the vertical axis is $1.93 \times 10^{-4}$ $\times \dot{m} \lambda^{5 / 2} r_{a}^{-9 / 4}\left(Z_{1} / A_{1}\right)^{3 / 8} ; \dot{m}$ is measured in $\mathrm{g} / \mathrm{sec}$, other units as in Fig. 3 . 


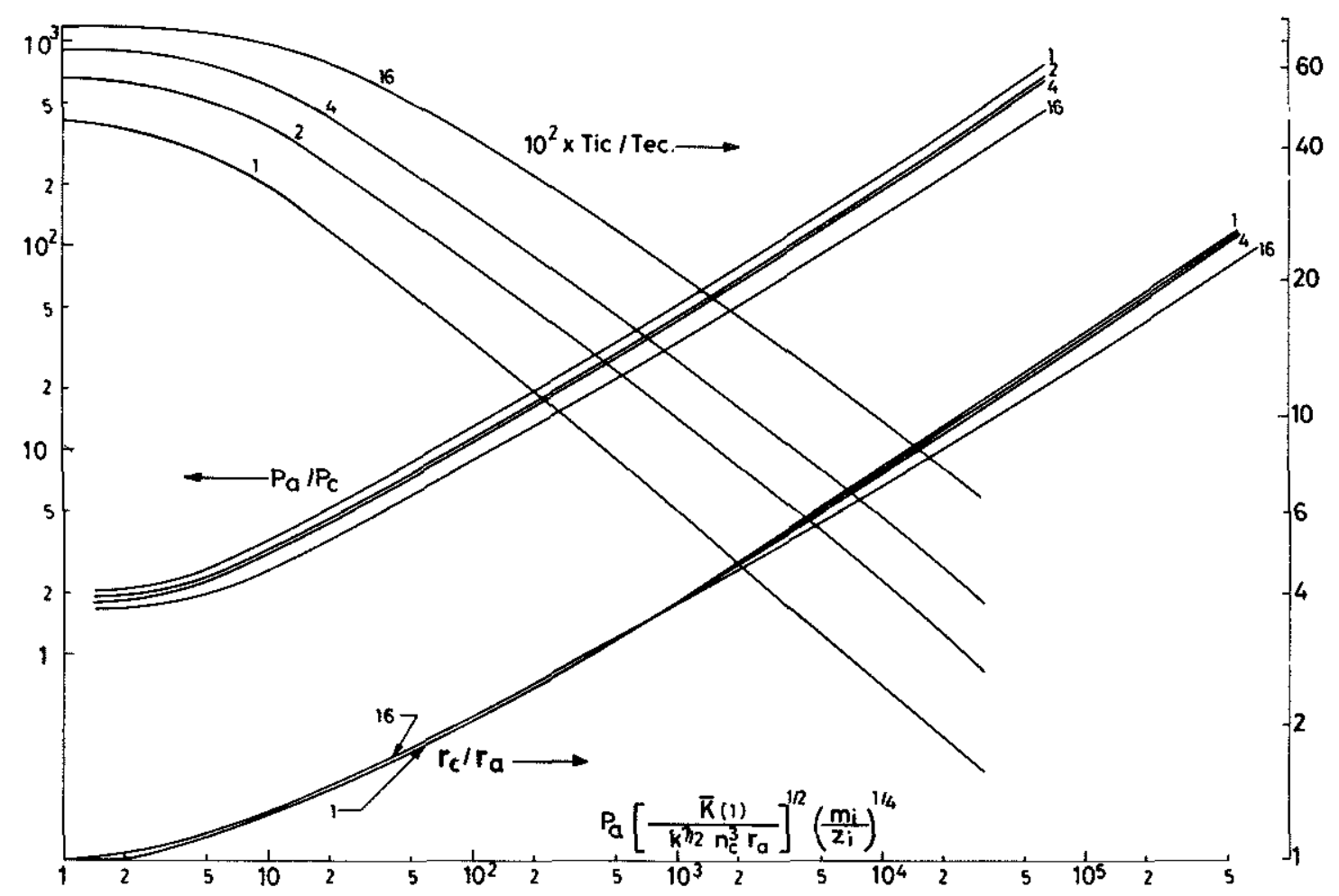

FIG. 5. Ion-to-electron temperature ratio at the critical radius, ablation to critical pressure ratio, and critical to ablation radius ratio, for $Z_{i}=1,2,4$, and 16 ; same horizontal axis as in Fig. 4.

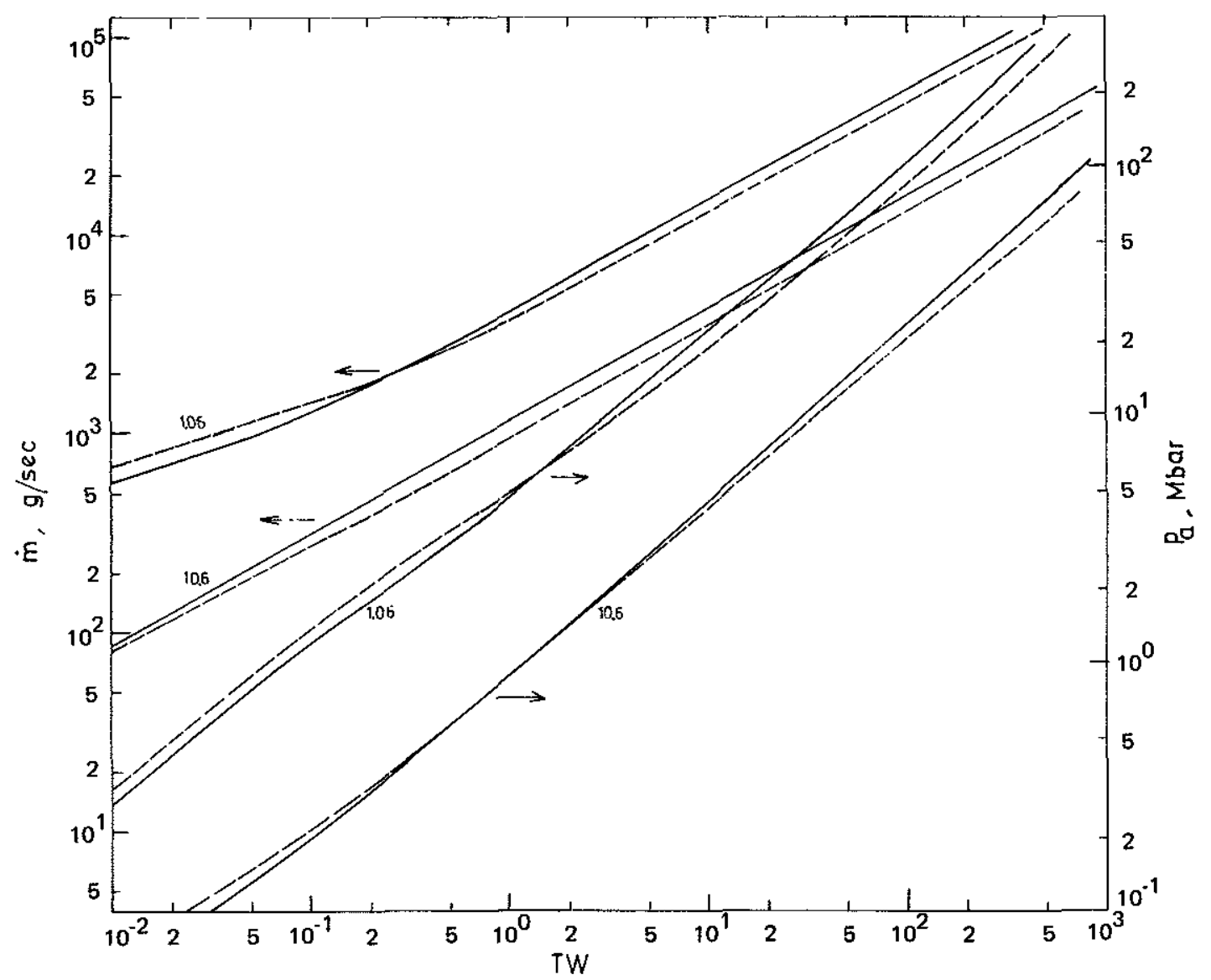

FIG, 6. Mass ablation rate and ablation pressure versus laser power for $r_{a}=500 \mu \mathrm{m}$, laser wavelengths $\lambda=1.06 \mu \mathrm{m}$ and $10.6 \mu \mathrm{m}, Z_{i}=1(-)$ and $16(---)$, and $A_{i} / Z_{1}=2 .\left(T W=10^{12} \mathrm{~W}\right)$. 


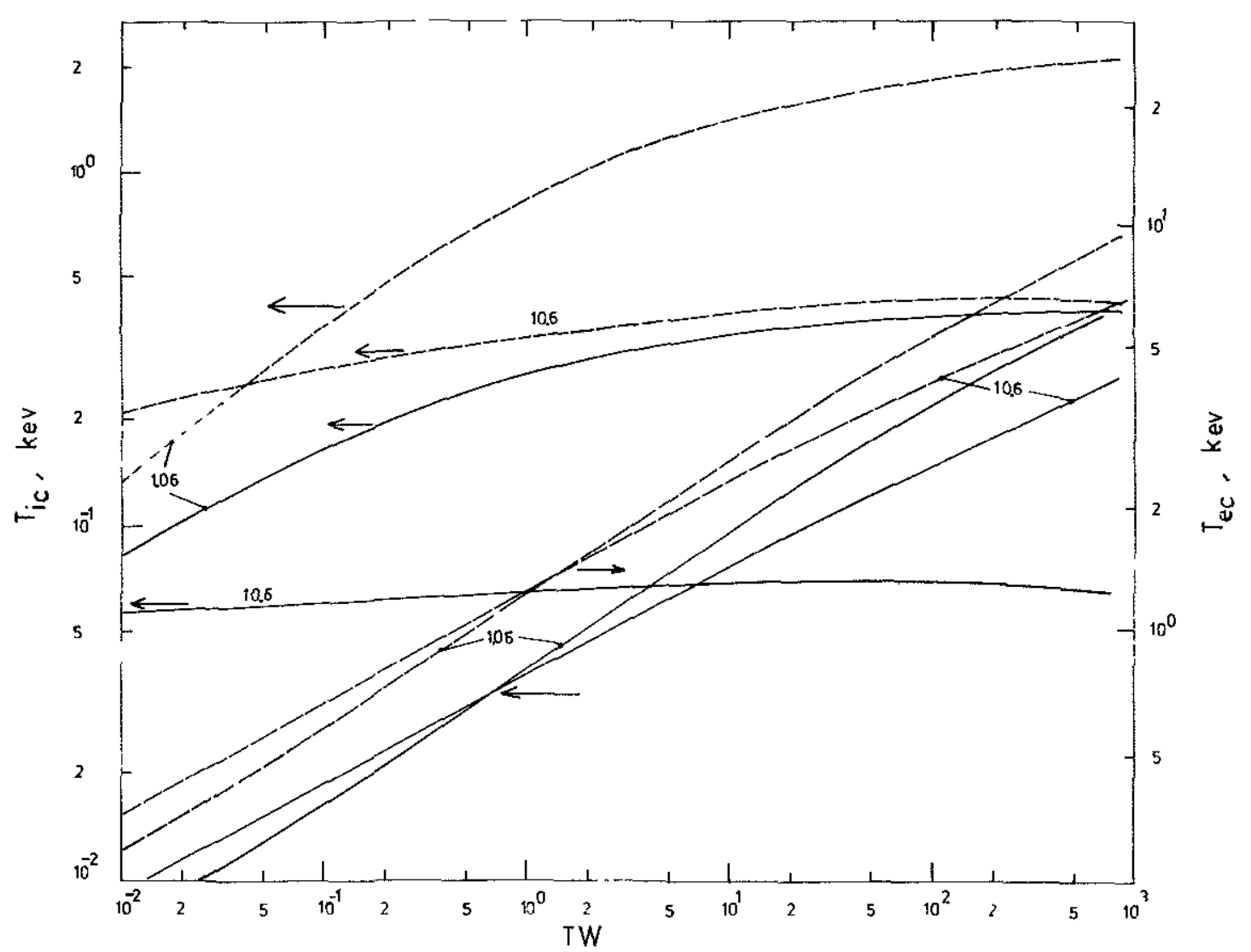

FIG. 7. Ion and electron temperatures at critical radius for the same conditions as in Fig. 6.

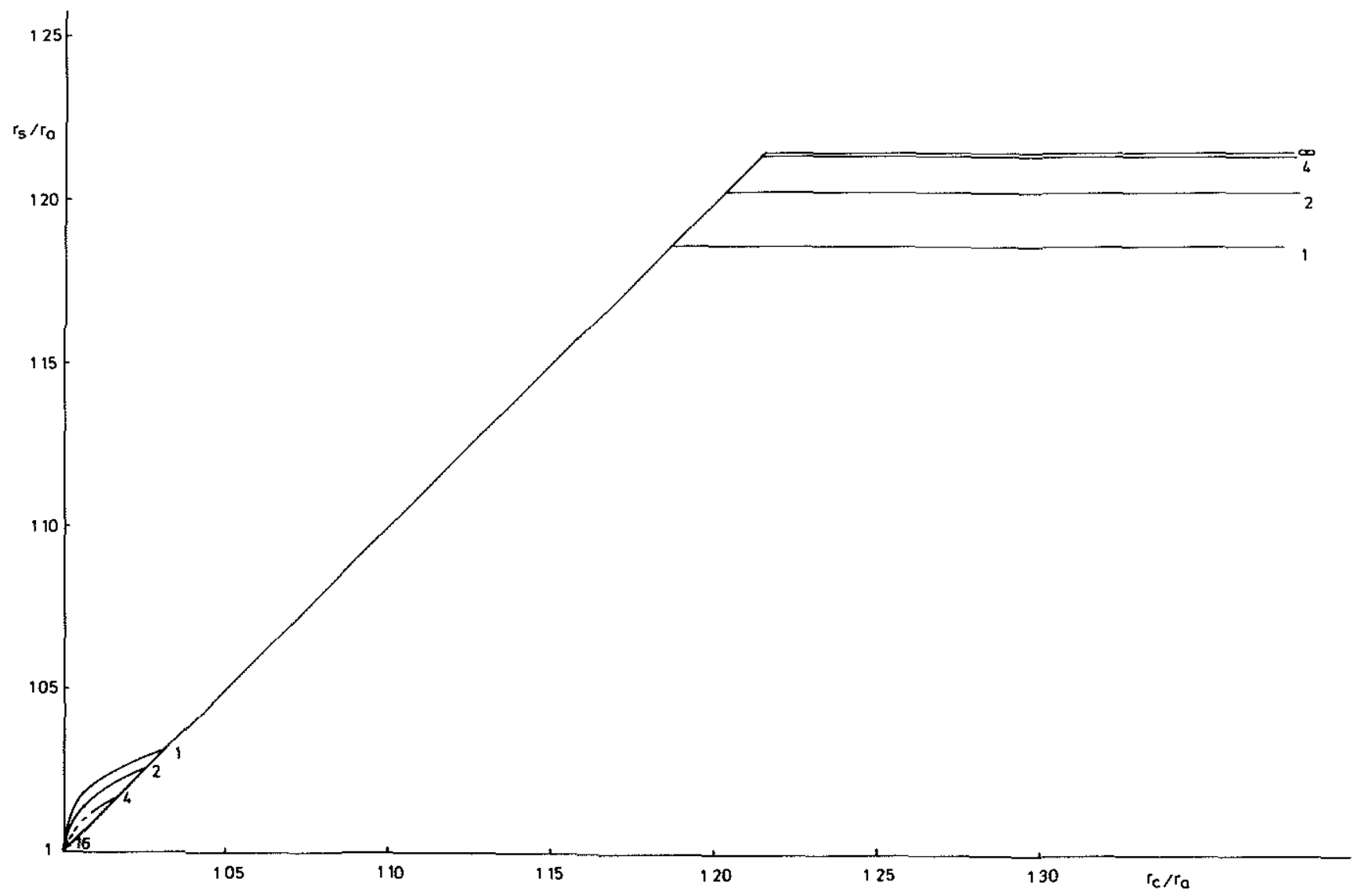

FIG. 8. Sonic radius versus critical radıus, for $Z_{i}=1,2,4,16$, and $\infty$. 
TABLE I. Parameters $\beta_{i}$ and $\eta_{c}^{*}$ for several values of ion charge number

\begin{tabular}{rll}
\hline$Z_{i}$ & $\beta_{i}$ & $\eta_{c}^{*}$ \\
\hline 1 & 146 & 180 \\
2 & 3.85 & 1.85 \\
4 & 6.54 & 195 \\
16 & 9.68 & 20 \\
$\infty$ & 11.30 & 2.05 \\
\hline
\end{tabular}

The flux-limit factor $f$ has been discussed in detail in the literature; while theory suggests that $f \simeq 0.6$, some experiments and numerical simulations could only be explained for values of $f$ sensibly smaller. ${ }^{9}$

Assume now $Z$, to be large; Eq. (8) decouples from (6) and (7), and they simplify into

$$
\begin{aligned}
& \frac{1}{2}\left(1-\frac{\theta_{e}}{u^{2}}\right) \frac{d u^{2}}{d \eta}=\frac{2 \theta_{c}}{\eta}-\frac{d \theta_{e}}{d \eta}, \\
& \frac{5}{2} \theta_{e}+\frac{1}{2} u^{2}=-\bar{q}+\bar{W} H(\eta) .
\end{aligned}
$$

Here $\bar{q}$ may take either one of two forms:

$$
\begin{aligned}
& \bar{q}_{c l} \equiv-\beta \eta^{2} \theta_{e}^{5 / 2} \frac{d \theta_{c}}{d \eta} \\
& \bar{q}_{s} \equiv-\left(\frac{m_{t}}{Z_{1} m_{e}}\right)^{1 / 2} f \frac{\theta_{e}^{3 / 2}}{u} \operatorname{sign}\left(\frac{d \theta_{e}}{d \eta}\right) .
\end{aligned}
$$

The solution to Eqs. (15) and (16) should satisfy obvious conditions: defining the function $\sigma \equiv \vec{q}_{c l} / \bar{q}_{s}$, we should have $\sigma<1$ in the regions where the classical flux was used, and $\sigma>1$ where the flux was saturated. Because of the continuity of the heat flux (except at $\eta_{c}$ ), we also required that $\sigma=1$ on the classical side of any boundary between classical and saturated regions.

The problem to be solved is similar to those in the previous section and in Ref. 7: Choosing $\eta_{c}$ freely in the range $(1, \infty)$, we must determine $u\left(\eta_{c}\right)$ and the eigenvalues $\beta$ and $\bar{W}$; then Eqs. (9)-(11) [where now $Z_{1}\left(Z_{\imath}+1\right)^{-1} \simeq 1$ ] will yield $\mu\left(\eta_{c}\right), P_{a}\left(\eta_{c}\right)$, and $W\left(\eta_{c}\right)$, from which $\mu\left(P_{a}\right)$ follows. It is worth stressing beforehand a few points proved in Ref. 4: (1) Both near $\eta=1$ and $\eta \rightarrow \infty$ heat conduction must be classical; (2) the Mach number $M=u / \theta_{e}^{1 / 2}$ at $\eta_{c}^{+}$cannot be less than unity (and grows with $\eta$ for $\eta>\eta_{c}$ ); and (3) if there is a saturated region within the interval $1<\eta<\eta_{c}$, Eq. (16) with $H=0$ and $\bar{q}=\bar{q}_{s}$ leads to $M=M_{s}$, where

$$
M_{s}\left(1+\frac{M_{s}^{2}}{5}\right)=\frac{2}{5}\left(\frac{m_{i}}{m_{e} Z_{i}}\right)^{1 / 2} f
$$

The last two points show that if $M_{1}<1$, there must exist a discontinuous jump from subsonic to supersonic flow.

Here, we only analyze the range $M_{s}>1$. Notice that $Z_{t}$ is some appropriate mean value of several ionization levels, which may be of order of 10 for typical ablators; we assumed $Z_{t} \gg 1$ (large ion mass number $A_{t}$ ) to simplify the analysis (the assumption $T_{i}=T_{e}$ of Ref. 4 leads to errors unless $Z_{i}$ is large). For the ablative regime of interest, and $A_{l}$ large, full ionization may not occur throughout the corona, the less so in the overdense, low-temperature, classical region at the boundary of which condition (17) is established: for $Z_{\text {, }}$ $\simeq A_{i} / 2, \quad Z_{1} \simeq A_{1} / 3, \quad$ and $Z, \quad \sim A_{i} / 4$, we get $f\left(M_{s}=1\right) \simeq 4.95 \times 10^{-2}, 4.04 \times 10^{-2}$, and $3.50 \times 10^{-2}$, respectively. Thus, the restriction $M_{s}>1$ corresponds roughly to condition $f>0.04$, allowing for most of the range $0.03<f<0.6$, considered in experiments and simulations. Furthermore if $M_{s}<1$ the problem that ensues is undetermined; in Ref. 4 the jump was assumed isothermal and an $a d$ hoc relation between densities, ahead and behind, was used. For $0.03<f<0.04\left(M_{s} \leqslant 1\right)$, that model does not appear better than just extrapolating results from the range $f>0.04$. On the other hand, if $M_{s}$ is small ( $f$ well below 0.04) the jump is large, implying substantial transformation from thermal into (macroscopic) kinetic energy; there are some doubts about the validity of such a model.

As shown in Sec. II, in the absence of a flux limit both $\beta$ and the solution $\left[u(\eta), \theta_{e}(\eta)\right]$ up to $\eta_{c}$, could be found, for any $\eta_{c}$ without recourse to the interval $\eta_{c}<\eta<\infty$. For $Z_{1}>46$, two $\eta_{c}$ intervals could be distinguished: As $\eta_{c}$ went from 1 to $\eta_{\mathrm{sl}}\left(\simeq 1.215\right.$ for $Z_{\mathrm{t}} \rightarrow \infty$, see Fig. 8$)$, the solution $\left[u(\eta), \theta_{e}(\eta)\right]$ changed, and $\beta$ went from zero to $\beta_{l}(\simeq 11.3$, for $\left.Z_{1} \rightarrow \infty\right)$, although $M\left(\eta_{c}\right) \equiv 1$. On the other hand, for $\eta_{c}>\eta_{s l}$ neither $\beta\left(=\beta_{l}\right)$, nor the solution (given in Fig. 2 of Ref. 7 for $Z_{1} \rightarrow \infty$, and called here $\left.\left[u_{0}(\eta), \theta_{0}(\eta)\right]\right)$ depended on $\eta_{c}$; defining $M_{0}(\eta)=u_{0} / \theta_{0}^{1 / 2}$, we had then $M\left(\eta_{c}\right)$ $=M_{0}\left(\eta_{c}\right)>1$.

Consider then any $f$ such that $M_{s}>1$ and let $\eta\left(M_{s}\right)$ be the root of the equation $M_{0}(\eta)=M_{s}$. If $1<\eta_{c}<\eta\left(M_{s}\right)$, neither $\beta$ nor the solution up to $\eta_{c}$, and in particular $u\left(\eta_{c}\right)$, are modified by the flux limit: for $1<\eta_{c}<\eta_{s l}$, use of (9) and (11) to eliminate $\eta_{c}$ yields $\mu\left(P_{a}\right)$, while for $\eta_{s l}<\eta_{c}$, Eq. (9) alone yields $\mu\left(P_{a}\right)$ because $\beta=\beta_{l}$. If $\eta\left(M_{\mathrm{r}}\right)<\eta_{c}$, the flux limit does not modify the solution up to $\eta\left(M_{s}\right)$, while $M=M_{s}$ and (17) hold for $\eta\left(M_{\mathrm{s}}\right)<\eta<\eta_{c} ; \beta=\beta_{l}$ and again (9) alone yields $\mu\left(P_{a}\right)$. Hence the relation $\mu\left(P_{a}\right)$ is independent of $f$ and is that given in Ref. 7.

To find $\bar{W}$ we must consider the interval $\eta_{c}<\eta<\infty$. We find that there are three different cases.

(a) Classical flux throughout the interval; as in Sec. II, $\bar{W}$ is found by requiring that $\theta_{\mathrm{a}^{2}} \rightarrow 0$ as $\eta \rightarrow \infty$.

(b) Saturated flux up to, say, $\eta_{c l}$, and classical for $\eta_{c l}<\eta<\infty ; \bar{W}$ is obtained by evaluating (16) at $\eta_{c}^{+}[H=1$, $\left.\vec{q}=\bar{q}_{s}=\left(m_{1} / m_{c} Z_{t}\right)^{1 / 2} f \theta_{e} / M\right]$ and then $\eta_{c l}$ is found by requiring $\theta_{e} \rightarrow 0$ as $\eta \rightarrow \infty$ [remember that for any tentative value of $\eta_{c l}$, the slope $d \theta / d \eta$ at $\eta_{c l}^{+}$follows from the requirement $\sigma\left(\eta_{c l}^{+}\right)=1$ ]

(c) Classical flux up to, say, $\eta_{f}$, saturated for $\eta_{f}<\eta<\eta_{c l}$, and classical again for $\eta_{c l}<\eta<\infty$. For $\bar{W}$ chosen within some range the solution, starting classical, saturates at a value $\eta_{J}(\bar{W})$ such that $\sigma=1$ at $\eta_{J}^{-}(\bar{W}) ; \bar{W}$ and $\eta_{f}$ are determined from the condition $d \sigma / d \eta=0$ at $\eta_{j}^{-}(\bar{W})$, and then $\eta_{c l}$ follows in the manner of (b). To justify the condition $d \sigma / d \eta=0$ at $\eta_{j}^{-}$, we evaluate the trivial equality

$$
\bar{q}_{s} \frac{d \sigma}{d \ln \eta}=\frac{d \bar{q}_{c l}}{d \ln \eta}-\sigma \frac{d \bar{q}_{s}}{d \ln \eta} \text { at } \eta_{f}^{-},
$$

using $\bar{q}_{c l}=\bar{W}-\theta_{e}\left(5+M^{2}\right) / 2, \bar{q}_{s}=\left(m_{t} / m_{e} Z_{t}\right)^{1 / 2} f \theta_{e} / M$, and Eq. (15) to obtain $d \bar{q}_{c l} / d \ln \eta$ and $d \bar{q}_{s} / d \ln \eta$ for $\eta<\eta_{f}$, and using (16) with $H=1$ and $\bar{q}=\left(m_{1} / Z_{1} m_{e}\right)^{1 / 2} f \theta_{c} / M$ and again (15), to obtain 
$\left.\frac{d \ln \theta_{e}}{d \ln \eta}\right|_{+}=4 \frac{\left(m_{t} / m_{e} Z_{t}\right)^{1 / 2} f-M^{3}}{3 M^{3}-5 M+\left(3 M^{2}-1\right)\left(m_{l} / Z_{t} m_{e}\right)^{1 / 2} f}$

We finally arrive at

$$
\begin{aligned}
\left.\bar{q}_{s} \frac{d \sigma}{d \ln \eta}\right|_{-}= & \frac{3 M^{3}-5 M+\left(m_{t} / Z_{t} m_{e}\right)^{1 / 2} f\left(3 M^{2}-1\right)}{2 M\left(M^{2}-1\right)} \\
& \times\left.\frac{d \theta_{e}}{d \ln \eta}\right|_{-}\left(\sigma^{+}-1\right) .
\end{aligned}
$$

Since $d \ln \theta_{e} / d \ln \eta l_{+}$should be negative, and $M \geqslant M_{s}>1$, the denominator in (18) is non-negative; then the left- and right-hand sides of (19) must be non-negative and nonpositive, respectively. Hence $\sigma^{+}=1$, and $d \sigma /\left.d \eta\right|_{-}=0$.

Figure 9 shows the correspondence between $f, W$ values and the different sequences of classical and saturated regions (for both $\eta<\eta_{c}$ and $\eta>\eta_{c}$ ). Figures 10 and 11 show $P_{a}$ and $r_{c}$ vs $W$ for several values of $f$.

\section{DISCUSSION}

\section{A. Arbitrary ion charge number}

If $Z_{i}$ is not large the ion temperature is coupled to other fluid variables. Fluid profiles for $Z_{t}=1$ are shown in Fig. 2 .
Complete results are given in Figs. 3-5 in dimensionless form. It is convenient to introduce the speed

$$
V\left(r_{a}, n_{c}, \frac{m_{\imath}}{Z_{t}}, Z_{t}\right) \equiv\left(\frac{r_{a} n_{c}}{m_{t} / Z_{t}} \frac{k^{7 / 2}}{\bar{K}\left(Z_{l}\right)}\right)^{1 / 4} .
$$

The horizontal axis of Fig. 3 is $W / r_{a}^{2} \rho_{c} V^{3}\left(Z_{t}=1\right)$ and that of Figs. 4 and 5 is $P_{a} / \rho_{c} V^{2}\left(Z_{1}=1\right)$; here $\rho_{c} \equiv n_{c} m_{i} / Z_{1}$. The vertical axis of Fig. 4 is $\dot{m} / 4 \pi r_{a}^{2} \rho_{c} V\left(Z_{1}=1\right), \dot{m}$ being the mass ablation rate $4 \pi m, \mu / Z_{i}$; the vertical axis of Fig. 3 is $\left[1.17 P_{a} / \rho_{c} V^{2}\left(Z_{t}=1\right)\right] /\left[W / 4 \pi r_{a}^{2} \rho_{c} V^{3}\left(Z_{t}=1\right)\right]^{2 / 3}$.

Results for particular conditions are given in Figs. 6 and 7 in dimensional form. As $Z_{1}$ goes from 1 to $16, \dot{m}$ and $P_{a}$ change at most by $25 \%$ and $30 \%$, respectively; $T_{e}$ and $T_{t}$ at $r_{c}$ change by about $60 \%$ and $600 \%$, respectively. Figures $3-$ 7 show that using $Z$ independent of position is an approximation that should hardly affect the results except for $T_{i}\left(r_{c}\right)$, and to some extent for $T_{e}\left(r_{c}\right)$ and $P\left(r_{c}\right)$.

Figure 8 shows the sonic radius $r_{s}$ versus the critical radius $r_{c}$. For $Z_{t}$ below some value $\left(Z_{t}<47\right)$ the flow at $r_{c}$ is subsonic if $r_{c} / r_{a}$ is close to unity. For $r_{c} / r_{a}$ such that $r_{c}>r_{s}$ (Fig. 8), Eq. (9) yields

$$
\dot{m} / 4 \pi r_{a}^{2} \rho_{c} V=\left(P_{a} / \rho_{c} V^{2}\right)^{5 / 6} Z_{i}^{5 / 6} /\left(1+Z_{i}\right)^{5 / 6} \beta_{l}^{1 / 6} \text {, }
$$

$\beta_{l}$ given in Table $\mathrm{I} ;\left[Z_{l}^{5} /\left(Z_{l}+1\right)^{5} \beta_{l}\right]^{1 / 6}$ ranges from 0.527

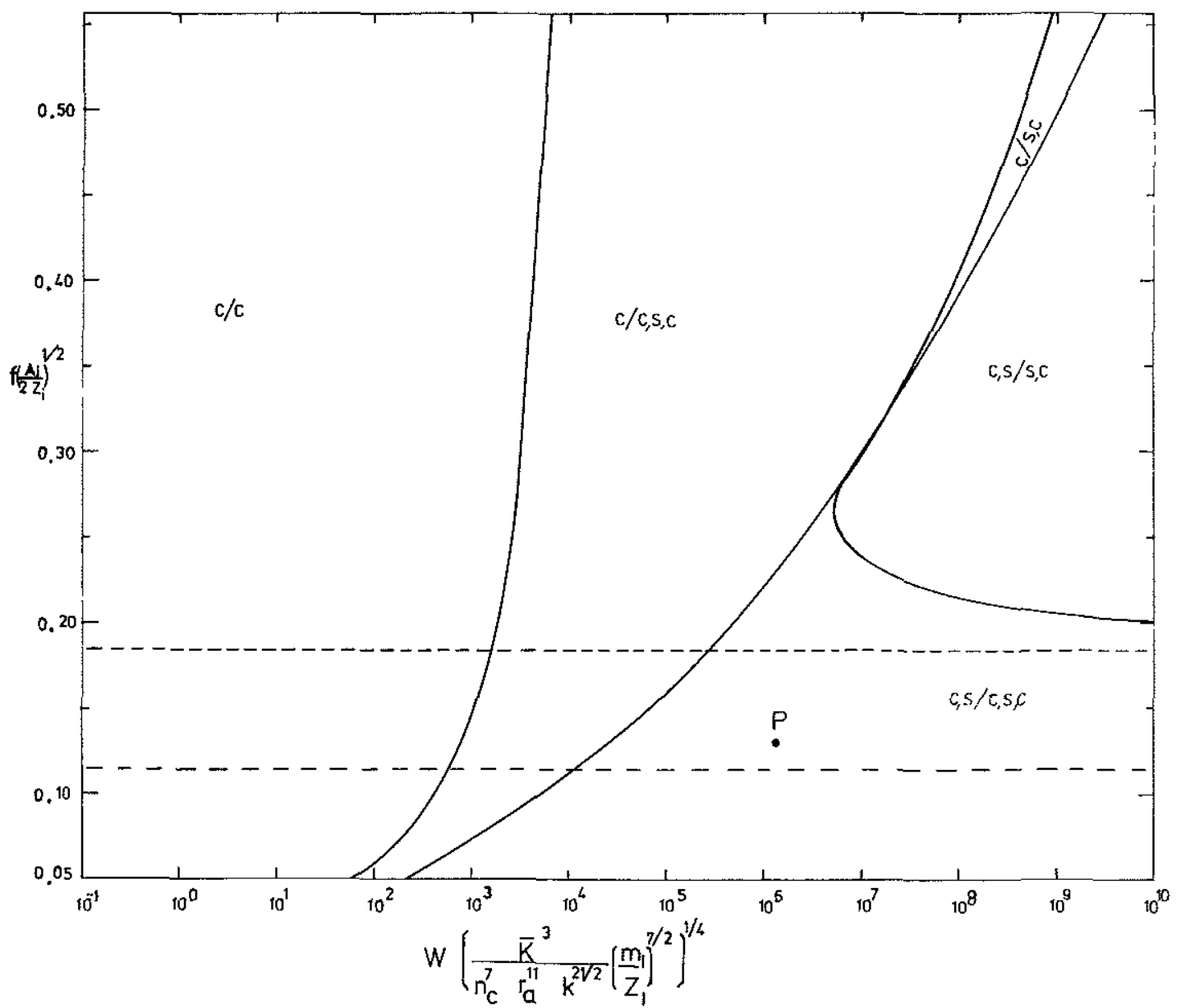

FIG. 9. Sequences of spatial regions with classical (c) and saturated (s) heat flux, in terms of flux limit $f$ and laser power $W$; horzontal axis differs from that in Fig. 3 by the factor $\left[\bar{K}\left(Z_{i}\right) / \bar{K}(1)\right]^{3 / 4}, \bar{K} \equiv$ Spitzer's heat-conductivity factor. For the conditions, for instance, of point $P$, the overdense flow presents two regions, classical next to $r_{a}$ and saturated next to $r_{c}$; the underdense flow is classical next to $r_{c}$, and far enough from the pellet, and saturated inbetween. The solidus marks the critical radius $r_{c} \cdot---M_{s}^{2}=5,-\cdots M_{s}^{2}=3$, in Eq. (17). 


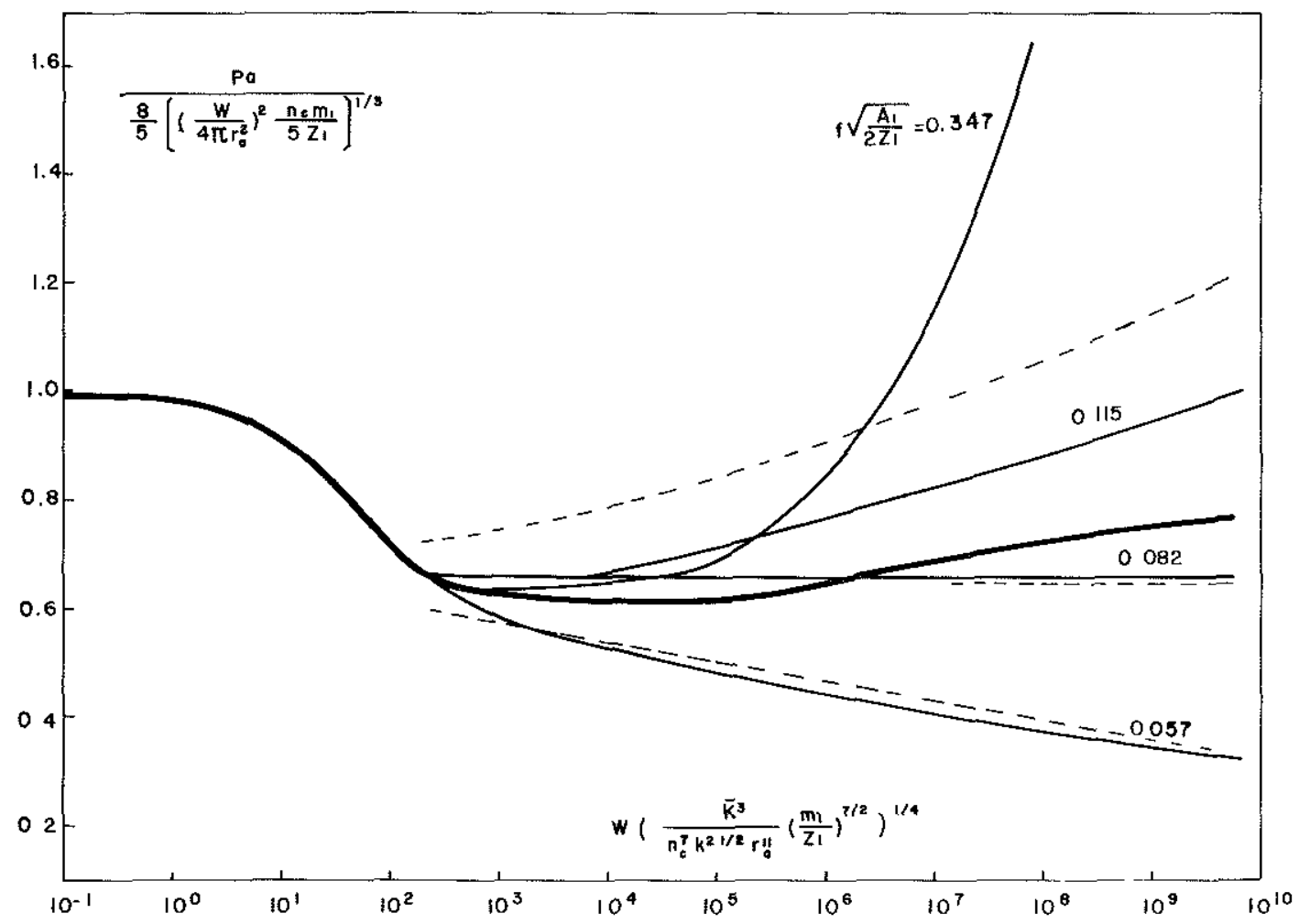

FIG. 10, $P_{a}$ vs $W$ for several values of $f$; results from Ref. 4 (-- -) and Ref. 7 (gross full line, no saturation considered) also shown. The vertical axis differs from that in Fig. 3 by factor $5^{4 / 3} / 8 \times 1.17$; same horizontal axis as in Fig, 9 .

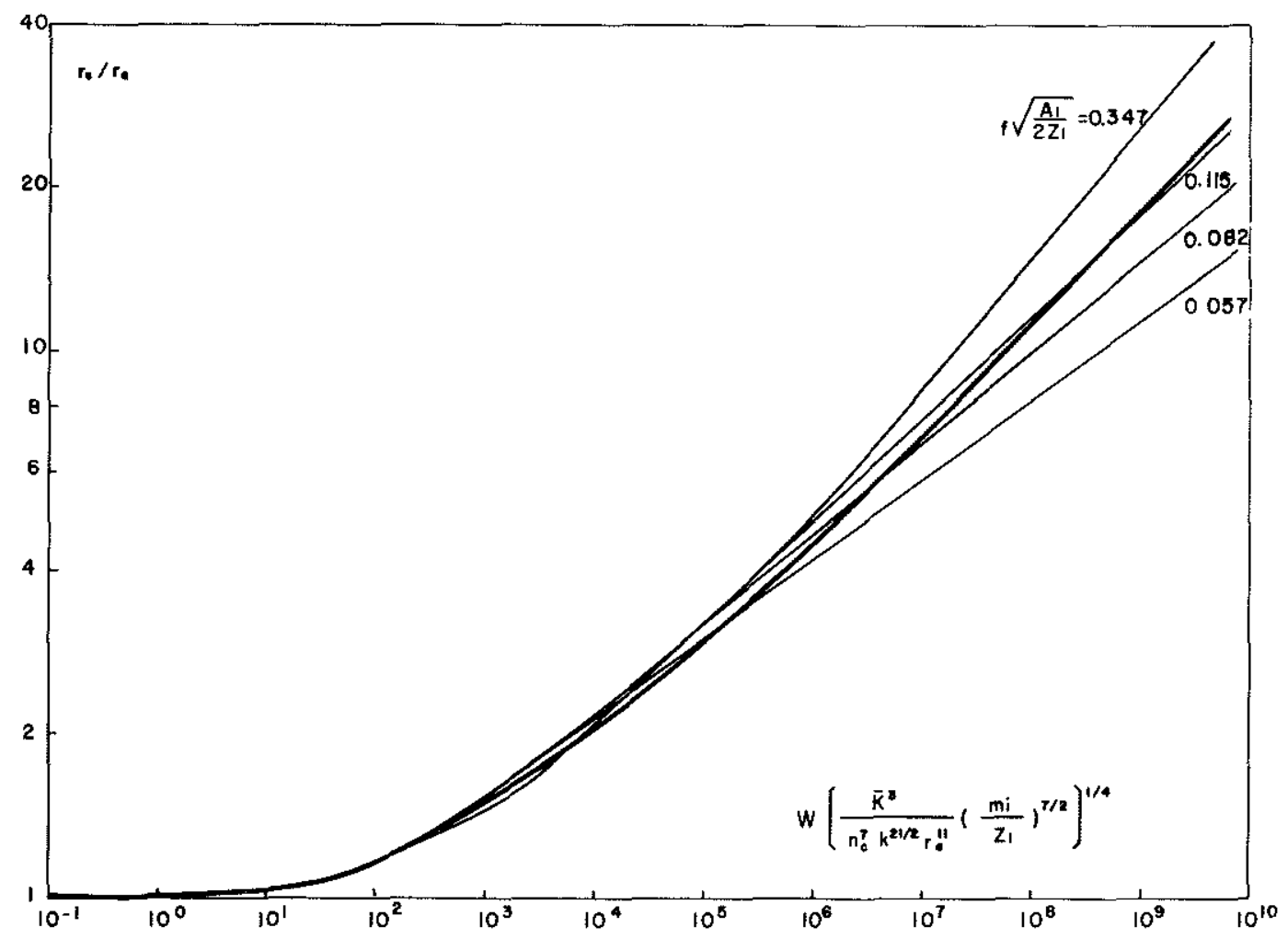

FIG. 11. Ratio $r_{c} / r_{a}$ vs $W$ for several values of $f$; results from Ref. 7 (gross full line) also shown. Same horizontal axis as in Fig. 9. 
at $Z_{i}=1$ to 0.668 at $Z_{i}=\infty$. For $r_{c} / r_{a} \rightarrow 1$ heat conduction is restricted to a thin deflagration layer. A rigorous analysis of such layers ${ }^{10}$ (detailed in Ref. 7 for $Z_{i} \rightarrow \infty$ ) gives

$$
\begin{aligned}
& \dot{m} / 4 \pi r_{a}^{2} \rho_{c} V=\left(5 n_{d} / 8 n_{c}\right)^{1 / 2}\left(P_{a} / \rho_{c} V^{2}\right)^{1 / 2}, \\
& P_{a} / \rho_{c} V^{2}=\left(128 n_{d} / 125 n_{c}\right)^{1 / 3}\left(W / 4 \pi r_{a}^{2} \rho_{c} V^{3}\right)^{2 / 3},
\end{aligned}
$$

where $n_{d}$ is the density on the underdense side of the layer; $\left(125 n_{c} / 128 n_{d}\right)^{1 / 3}$ ranges from 1.17 at $Z_{i}=1$ to $5^{4 / 3} / 8$ at $Z_{i}=\infty$ (Ref. 10).

Results in Ref. 2, which only apply for $r_{c}>r_{s}$, were given in terms of values of physical variables at $r_{s}$. To conveniently relate those results to ours we now give the relevant sonic values, from our analysis,

$$
\begin{aligned}
& r_{s} / r_{a}=1.215, \quad P_{s} / \rho_{c} V^{2}=\left(\theta_{s}^{1 / 2} r_{a}^{2} / r_{s}^{2}\right) P_{a} / \rho_{c} V^{2}, \\
& Z_{i} k T_{e s} / m_{i} V^{2}=\theta_{s} \beta l^{1 / 3}\left(P_{a} / \rho_{c} V^{2}\right)^{1 / 3}, \\
& n_{s}=P_{s} / k T_{e s},
\end{aligned}
$$

$\beta_{l} \simeq 11.3, \theta_{s} \simeq 0.42$ (Fig. 2 , Ref. 7 ); $P_{a} / \rho_{c} V^{2}$ can be obtained from Fig. 10 (gross full line). To write the preceding equations we assumed $Z_{i}$ large, for which case the hypothesis $T_{i}=T_{e}$ of Ref. 2 is unimportant (except for the determination of $T_{i}$ ). The parameter " $M$ " introduced in Ref. 2 should be set equal to $2 / 3$.

\section{B. Heat flux saturation}

To analyze saturation we considered $Z_{i}$ large. Figure 9 shows the sequence of spatial regions with classical and saturated flux, in terms of the dimensionless parameters of the problem, $f\left(A_{i} / 2 Z_{i}\right)^{1 / 2}$ and $W / r_{a}^{2} \rho_{c} V^{3} ;$ Fig. 10 shows $P_{a}(f, W)$.

Equation (17) can be written as $2 \phi=M_{s}\left(1+M_{s}^{2} / 5\right)$, $\phi \simeq 12.1 f\left(A_{i} / 2 Z_{t}\right)^{1 / 2}$, a quantity used in Ref. 4. It was assumed there that $M_{s}^{2} / 5 \ll 1$, while we considered $M_{s}^{2}>1$; thus, the analyses are somehow complementary. Actually in Ref. 4 , its analysis was assumed valid up to $\phi \simeq 1\left[M_{s}^{2}=2\right.$, $\left.f\left(A_{i} / 2 Z_{i}\right)^{1 / 2} \simeq 0.082\right]$; our Fig. 10 confirms this. Agreement with simulations was found up to $\phi=1.4^{4}\left[M_{s}^{2}=3\right.$, $\left.f\left(A_{i} / 2 Z_{i}\right)^{1 / 2} \simeq 0.115\right]$, where we find some disagreement (Fig. 10). Results from Ref. 4 should not be used above $M_{s}^{2}=3$, marked in Fig. 9. They should not be used either for $W$ too low $\left(r_{c} / r_{a}\right.$ too close to unity): Fig. 9 shows that the sequence $(c, s / c, s, c)$ (see caption to Fig. 9 for symbols explanation), considered in Ref. 4 for $M_{s}^{2}<5$, has a restricted range of validity. [For $M_{s}^{2}>5$, Ref. 4 discussed only the sequence $(c, s / s, c)$.] To conclude we note that recent spherical experiments ${ }^{11}$ suggest $f$ values higher than those found in planar geometry.

The result for $P_{a}(W)$ obtained when saturation is not considered ${ }^{7}(f \rightarrow \infty)$ is also drawn in Fig. 10 which shows that for a given power $W$ saturation may decrease (low $f$ ) or increase (high $f$ ) the pressure $P_{a}$ relative to the value attained when $f \rightarrow \infty$. The explanation of this result lies in a fact recalled in Sec. I: for $f \rightarrow \infty$ and $r_{c} / r_{a}$ large most of the energy absorbed is lost by outward conduction. ${ }^{7}$ Hence, for high $f$ (weak flux inhibition) only this huge outward loss is inhibited, increasing $P_{a}$, while for $f$ low enough the inward heat flux is strongly inhibited, reducing $P_{a}$. This leads to a second, seemingly paradoxical effect, seen in Fig. 10, too: In some cases a decrease in $f$, with $W$ fixed, affects more strongly fux inhibition in the underdense flow, resulting in a higher $P_{a}$ (or a lower $W$ for given pressure).

Figure 10 shows that for some values of $f$,

$$
P_{a} /\left[\left(W / r_{a}^{2}\right)^{2} n_{c} m_{i} / Z_{i}\right]^{1 / 3} \simeq \text { const. }
$$

This is not a trivial result, required by dimensional arguments; the left-hand side of (22) could have been any function of the abscissa, ${ }^{12}$ or alternatively, of the ratio $r_{c} / r_{a}$ [for instance, $r_{c}$ could figure instead of $r_{a}$ in (22)]. Nuckolls, in fact, suggested $^{6} P_{a}\left[\left(W / r_{a}^{2}\right)^{2} n_{c} m_{i} / Z_{i}\right]^{-1 / 3} \simeq\left(r_{c} / r_{a}\right)^{2 / 3}$, which would only be right for $f$ high enough. In Ref. 4, emphasis was laid on low values of $f$, for which the left side of (22) decreases with growing $W$.

Figure 11 shows that $r_{c} / r_{a}$ is weak function of the fluxlimit factor. We also find that $P_{a} / P_{c}$ and $T_{i c} / T_{e c}$ decrease with $f$, and that the relation $\dot{m}\left(P_{a}\right)$ is not affected by saturation. We had found earlier [Eq. (21)] that for $r_{c}>r_{s}, \dot{m}\left(P_{a}\right)$ was independent of $n_{c}$ and nearly so of $Z_{i}$ : $\dot{m} \propto\left(A_{i} / Z_{i}\right)^{7 / 12} r_{a}^{11 / 6} P_{a}^{5 / 6}$. Since $r_{a}(t)$ will follow from $\dot{m}\left(r_{a}, P_{a}\right)$ and the pressure law $P_{a}(t)$, once this law is selected the ablation rate $\dot{m}(t)$ appears to be unaffected by conditions in the corona.

When the analysis of Ref. 4 is valid, the parameter $\hat{\sigma}$ introduced there is approximately $1.3 \times 10^{-4} \phi^{-7 / 3}(\mathrm{~W} /$ $\left.r_{a}^{2} \rho_{c} V^{3}\right)^{4 / 3}$. Note that the ratio $W / r_{a}^{2} \rho_{c} V^{3}$ (horizontal axis of Figs. 9-11) covers a very large range of values; for $1000>r_{a}(\mu \mathrm{m})>50$ (beginning and end of compression), $0.35<\lambda(\mu \mathrm{m})<10.6$ (third harmonic of $\mathrm{Nd}$ and $\mathrm{CO}_{2}$ ), $Z_{i}=10, A_{i} \simeq 2 Z_{i}$, and a single power $\left(10^{13} \mathrm{~W}\right)$ whose ratio ranges from 40 to $2.3 \times 10^{10}$. The influence of inverse bremsstrahlung for low values of $W / r_{a}^{2} \rho_{c} V^{3}$, and hot electrons for high values is considered elsewhere. ${ }^{13}$

\section{ACKNOWLEDGMENTS}

This research is partly based on a doctoral thesis by $\mathrm{J}$. Sanz, and was performed under the auspices of the Junta de Energía Nuclear of Spain.

${ }^{1}$ A. Caruso, Plasma Phys. 18, 241 (1976).

${ }^{2}$ S. J. Gitomer, R. L. Morse, and B. S. Newberger, Phys. Fluids 20, 234 (1977).

${ }^{3}$ Yu. V. Afanas'ev, E. G. Gamalii, O. N. Krokhin, and V. B. Rozanov, Zh. Eksp. Teor. Fiz. 71, 594 (1976) [Sov. Phys. JETP 44, 311 (1977)].

${ }^{4}$ C. E. Max, C. F. McKee, and W. C. Mead, Phys. Fluids 23, 1620 (1980).

${ }^{5}$ L. L. Cowie and C. F. McKee, Astrophys. J. 211, 135 (1977).

${ }^{6} \mathrm{~J}$. H. Nuckolls, in Laser Interaction and Related Plasma Phenomena, edited by H. Schwarz and H. Hora (Plenum, New York, 1974), Vol. 3B, p. 399.

7J. Sanz, A. Liñán, M. Rodríguez, and J. R. Sanmartín, Phys. Fluids 24, 2098 (1981).

${ }^{8}$ As in Ref. 7, we find that there is a value $\eta_{c}^{*}$ such that, at large values of $\eta$, $\theta_{e}$ behaves as $\eta^{-4 / 3}, \eta^{-2 / 5}$, and $\eta^{-2 / 7}$ for $\eta_{c}$ less than, equal to, and greater than $\eta_{\mathrm{r}}^{*}$, respectively.

${ }^{9}$ W. Kruer, Comments Plasma Phys. 5, 69 (1979).

${ }^{10}$ J. R. Sanmartín and A. Barrero, Phys. Fluids 21, 1957 (1978).

${ }^{11}$ T. J. Goldsack, J. D. Kilkenny, B. J. MacGowan, P. F. Cunningham, C. L. S. Lewis, M. H. Key, and P. T. Rumsby, Phys. Fluids 25, 1634 (1982).

${ }^{12}$ Actually dimensional arguments would introduce a second dimensionless parameter, $n_{c} r_{a}^{3}$, which, however, appears nowhere in the results.

${ }^{13}$ J. R. Sanmartín, R. Ramis, J. L. Montañés, and J. Sanz (to be published), J. A. Nicolas and J, R. Sanmartín (to be published). 